Anton A. Kiss, Edwin Zondervan, Richard Lakerveld, Leyla Özkan (Eds.)

Proceedings of the $29^{\text {th }}$ European Symposium on Computer Aided Process Engineering

June $16^{\text {th }}$ to $19^{\text {th }}$, 2019, Eindhoven, The Netherlands. (C) 2019 Elsevier B.V. All rights reserved.

\title{
Improving the prediction of multi-component tablet properties from pure component parameters
}

\author{
Hikaru G. Jolliffe ${ }^{\mathrm{a}^{*}}$, Foteini Papathanasiou ${ }^{\mathrm{b}}$, Elke Prasad ${ }^{\mathrm{a}}$, Gavin Halbert ${ }^{\mathrm{a}}$, John \\ Robertson ${ }^{\mathrm{a}}$, Cameron J. Brown ${ }^{\mathrm{a}}$, Alastair J. Florence ${ }^{\mathrm{a}}$. \\ ${ }^{a}$ EPSRC Centre for Innovative Manufacturing in Continuous Manufacturing and \\ Crystallisation, University of Strathclyde, Technology and Innovation Centre, 99 \\ George Street, Glasgow G1 1RD, United Kingdom. \\ ${ }^{b}$ Strathclyde Institute of Pharmacy \& Biomedical Sciences, University of Strathclyde, \\ 161 Cathedral St, Glasgow G4 ORE, United Kingdom.
}

hikaru.jolliffe@strath.ac.uk

\begin{abstract}
Direct compaction tabletting, a widely used secondary downstream processing operation, has recently received significant research attention. Experimental data can be used to fit model parameters for the prediction of single-component tablet thickness and hardness with good agreement, and this has been done for two components (Avicel ${ }^{\circledR} \mathrm{PH}-101$ and Pharmatose $\left.{ }^{\circledR} 50 \mathrm{M}\right)$. These pure component parameters have then been used to predict multicomponent tablet properties, with the use of novel modified parameter averaging calculations improving predictions. Furthermore, a relation has been developed to estimate the required gap between tablet press punch faces for multicomponent tablets based on the gaps required for pure components; a quick and efficient way to estimate the necessary equipment settings to generate the desired compaction forces is a useful tool.
\end{abstract}

Keywords: direct compaction, tablet prediction, optimisation.

\section{Introduction}

Continuous Pharmaceutical Manufacturing (CPM) is a promising alternative to the current paradigm of batch production, and mathematical modelling and simulation is a useful tool within the methodology of CPM (Lee et al., 2015). The final step in many production process is final product formulation. In the case of solid dosage forms such as tablets the critical quality attributes include tablet tensile strength and thickness; appropriate dissolution rates are also crucial (Velasco et al., 1999). Currently, timeconsuming design-of-experiment (DOE) approaches are commonly used to determine the necessary conditions to achieve the required tablet properties, and the need for costeffective R\&D methodologies brings process modelling and simulation to the forefront of initial stages of process option evaluation (Diab and Gerogiorgis, 2018).

In the present work, we predict the properties of multicomponent tablets using parameters determined from pure component compaction data; a model by Gavi and Reynolds (2014) has been used to fit the parameters for tablet compaction. The use of pure component parameters in the prediction of multicomponent tablet properties entails the use of some form of mixing or averaging rule, and the appropriateness of currently implemented rules are analysed, with novel modifications made where necessary. 


\section{Compaction experiments}

Experiments (Papathanasiou, 2018) were performed for varying tablet weights (200, 250 or $300 \mathrm{mg}$ ) and components (microcrystalline cellulose: Avicel ${ }^{\circledR} \mathrm{PH}-101$; $\alpha$-lactose monohydrate: Pharmatose $\left.{ }^{\circledR} 50 \mathrm{M}\right)$. Tablet thickness and hardness were recorded for a range of compaction forces. Tablet masses were also measured after ejection (due to material flowability, there was always some variation from target tablet mass, approximately $\pm 2 \%$ ). For each compaction force, experiments were performed 10 times (i.e. 10 tablets were produced). In addition to the above experiments being done for pure components, a similar set of experiments were done for binary tablets of lactose and cellulose, to allow the assessment of binary tablet predictions.

The pure component experiments showed the expected trends, such as hardness increasing with increasing compaction force, and thickness decreasing with increasing compaction force (routine data not shown due to space constraints - available in the work of Papathanasiou, 2018). Cellulose achieves significantly higher hardness values (up to $450 \mathrm{~N}$ for $250 \mathrm{mg}$ tablets) than lactose (up to approximately $50 \mathrm{~N}$ ), which is unsurprising as Avice ${ }^{\circledR} \mathrm{PH}$ was introduced with direct compaction in mind. The hardness values achieved with the mixtures fall between those of pure cellulose and pure lactose, as one might expect.

\section{Compaction model}

The compaction model used here is one developed by Gavi and Reynolds (2014), and is also included in the FormulatedProducts module of the gPROMS software package (henceforth called gFormulate), produced by Process Systems Enterprise (PSE, 2018). The model has several key equations. The first computes the tablet relative density $\rho^{*}$ (Equation 1). Here, $\rho_{0}^{*}$ is the relative density at zero compaction pressure (taken to be the tapped density of the powder), $P$ is compaction pressure (MPa) and $K$ is the compressibility constant (a dimensionless fitting parameter). Tablet $\rho^{*}$ values can be calculated from the compressed tablet densities and the true density of the material ( $\rho^{\text {crys }}$, determined experimentally with a gas pycnometer). Equation 1 is used first in the calculations - the model is sequential. With relative density calculated, tablet thickness $h$ is then calculated for known $\rho^{\text {crys }}$, mass $M$, and diameter $d$ (Equation 2; assumes flatfaced cylinders) (Fell and Newton, 1970).

Another key equation is that governing tensile strength, $\sigma$ (Equation 3). Here, $\sigma_{0}$ is the tensile strength at zero porosity (i.e. a fitting parameter corresponding to the theoretical maximum possible compaction, units of $\mathrm{MPa}$ ), $k_{b}$ is the bonding capacity (also a dimensionless fitting parameter), and $\varepsilon$ is tablet porosity (Equation 4). The three key parameters, then, are the compressibility constant $(K)$, the tensile strength at zero porosity $\left(\sigma_{0}\right)$ and bonding capacity $\left(k_{b}\right)$. Experimentally, tensile strength can be calculated from hardness via Equation 5, where $F$ is the compaction force in $\mathrm{kN}$.

$$
\begin{aligned}
& \rho^{*}=\rho_{0}^{*} P^{1 / K} \\
& h=\frac{M}{\rho^{*} \rho^{c r y s}} \frac{4}{\pi d^{2}}
\end{aligned}
$$


$\sigma=\sigma_{0} e^{-k_{b} \varepsilon}$

$\varepsilon=1-\rho^{*}$

$\sigma=\frac{2}{\pi} \frac{F}{h d}$

\section{Pure component parameter estimation}

Comprehensive experimental data allows for parameters to be fitted for use in empirical and data-driven models. In this work, the parameters being fitted are compressibility constant $(K)$, tensile strength at zero porosity $\left(\sigma_{0}\right)$ and bonding capacity $\left(k_{b}\right)$.

The pure component experimental data were imported into gFormulate, where a digital flowsheet of the process was created. The control variables are compaction pressure $P$ and tablet weight $M$, while the key measured variables are tablet thickness $h$ and hardness $H$ (the diametrical load which causes tablet failure). With three fitted parameters and four variables (two control, two measured), the problem was straightforward, and computationally undemanding; the parameters themselves (for cellulose/Avicel ${ }^{\circledR} \mathrm{PH}-101$ and lactose/Pharmatose ${ }^{\circledR} 50 \mathrm{M}$ ) are given in Table 1 .

\section{Multi-component tablet property prediction}

Pure component parameters that reliably predict single-component tablets have been determined. The subsequent use of these for multi-component tablet property prediction has been investigated, which entails the use of some form of averaging or mixing rule for the model parameters $K, \sigma_{0}$ and $k_{b}$. Doing this by pre-compaction component volume fractions is one option (Equation 6) (Gavi and Reynolds, 2014). Results for mixtures of lactose-cellulose indicate that while predictions for hardness are good at lower compaction forces, there is a trend of over-prediction which becomes more evident at higher compaction forces (Fig. 1). However, this trend is not present for pure components, i.e. pure lactose and pure cellulose tablets are predicted reasonably well, with no significant hardness over-prediction at higher compaction forces (Fig. 1B). This implies the potential for prediction improvement from using alternative parameter averaging/mixing rules.

The volume fractions of the components after compaction (as opposed to prior) can be estimated by assuming they compact at similar rates as when they are a pure component (i.e. a $25 \%$ volume reduction for pure material $i$ when subjected to a given force implies a $25 \%$ reduction for component $i$ when a mixture is subjected to the same force), and can be computed via Equation 7 (Reynolds et al., 2017).

Table 1. Optimal pure component parameter values for Avicel ${ }^{\circledR}$ PH-101 and Pharmatose ${ }^{\circledR} 50 \mathrm{M}$.

\begin{tabular}{|c|c|c|c|c|c|c|}
\hline \multirow{2}{*}{ Component } & \multicolumn{2}{|c|}{$\begin{array}{l}\text { Tensile strength } \\
\text { at zero porosity }\end{array}$} & \multicolumn{2}{|c|}{$\begin{array}{l}\text { Bonding } \\
\text { capacity }\end{array}$} & \multicolumn{2}{|c|}{$\begin{array}{c}\text { Compressibility } \\
\text { constant }\end{array}$} \\
\hline & $\sigma 0(\mathbf{M P a})$ & $99 \%$ CI & $k_{b}(-)$ & $99 \% \mathrm{CI}$ & $K(-)$ & $99 \% \mathrm{CI}$ \\
\hline Avicel ${ }^{\circledR} \mathrm{PH}-101$ & 12.0067 & \pm 1.9080 & 7.5847 & \pm 0.6362 & 4.2008 & \pm 0.1372 \\
\hline Pharmatose ${ }^{\circledR} 50 \mathrm{M}$ & 1.6789 & \pm 0.3212 & 11.6020 & \pm 0.8704 & 9.0901 & \pm 0.2130 \\
\hline
\end{tabular}




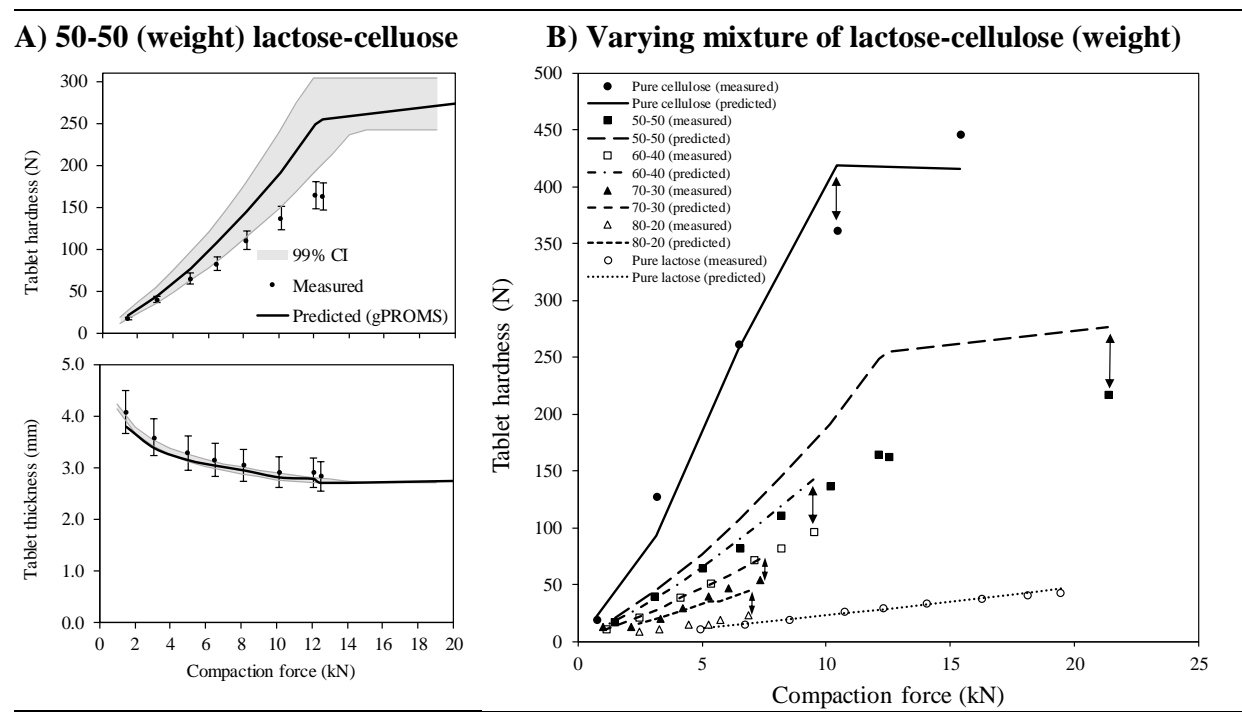

Figure 1. Predicted (Gavi and Reynolds, 2014) vs measured $250 \mathrm{mg}$ tablet hardness and thickness for varying mixtures of lactose-cellulose. For each substance, the predicted curves use the same respective set of parameters. The parameters can be found in Table 1. Arrows in B are a visual aid, to indicate which predicted curve corresponds with which experimental data set.

$$
\begin{gathered}
p_{\text {mix }}=\sum_{i \in C} \varphi_{i} p_{i}, \quad p=\left\{K, \sigma_{0}, k_{b}\right\} \\
\varphi^{\prime}{ }_{i}=\frac{m_{i} /\left(1-\varepsilon_{i}\right) \rho_{i}^{c r y s}}{\sum_{i \in C} m_{i} /\left(1-\varepsilon_{i}\right) \rho_{i}^{c r y s}} \\
\sigma_{T a b}=\prod_{i \in C}{\sigma_{i}}^{\varphi^{\prime}{ }_{i}}
\end{gathered}
$$

The Reynolds et al. (2017) model then use Equation 8 to compute the tensile strength of multicomponent tablets. In essence, tensile strengths for each component are calculated on a pure basis, then averaged using Equation 8. However, this approach relies on the components being of similar particle size (Reynolds et al., 2017). For applications where the particle sizes are different, such as in this work (Avicel ${ }^{\circledR} \mathrm{PH}-101$ has an average particle size of $50 \mu \mathrm{m}$, Pharmatose ${ }^{\circledR} 50 \mathrm{M}$ has an average size of $360 \mu \mathrm{m}$ ), an alternative approach is required.

The approach used in this work is to use Equation 6 to average the compressibility constant $K$ as before, then use Equation 7 to compute component volume fractions in the compact $\left(\varphi_{i}^{\prime}\right)$, and then use these volume fractions to average the post-compaction tensile strength $\left(\sigma_{0}\right)$ and bonding capacity $\left(k_{b}\right)$ parameters (Equation 9$)$ for use with Equation 3.

$$
\sigma_{0 m i x}=\sum_{i \in C} \varphi^{\prime}{ }_{i} \sigma_{0 i}, \quad k_{b m i x}=\sum_{i \in C} \varphi_{i}^{\prime} k_{b i}
$$




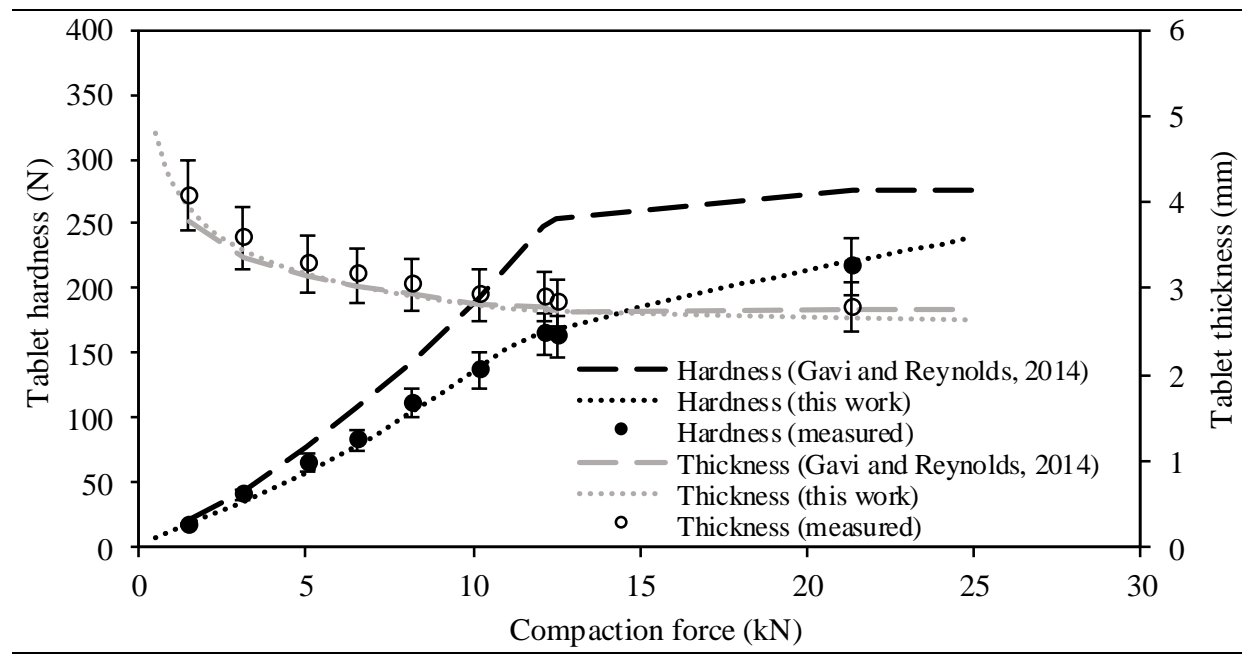

Figure 2. Predicted vs measured (experimental) $250 \mathrm{mg}$ tablet hardness and thickness for $250 \mathrm{mg}$ lactose-cellulose tablets (50-50 by weight), illustrating prediction improvements from using postcompaction volume fractions to weight the pure component parameters.

This approach results in improved predictions (Fig. 2). Compared with the previous method (Fig. 1, Fig. 2 dashed lines) the improvements are evident, especially for tablet hardness. While predictions using the previous method agreed with measured data at lower compaction forces, the method outlined in here produces predictions which match the experimental data across the entire range of compaction forces.

The main variable in the compaction model used here is, as with many compaction models, the pressure applied (or the force in some cases) (Equation 1). However, in tablet presses there frequently is not a setting or dial for pressure, but a way to set the distance to which the punch faces compact the material (punch gap, PG); knowing what pressure or force is required might not be immediately useful to a user in the lab. We have taken the compaction force and PG data, and have been able to determine a relation between them, adjusted for tablet weight. An example of this for cellulose is given in Fig. 3A. This variable, which we have called the gap-mass factor $\gamma$, has units of $\mathrm{mm} / \mathrm{g}$. When plotted against compaction pressure as in Fig. 3A, a power law relation (Equation 10) can be determined for the curved portion corresponding to lower compaction pressures; the horizontal portion, corresponding to the limit of zero porosity, is less useful as there is no improvement in tablet properties at these compaction pressures. This fitting of the curved portion will result in two parameters $a$ and $b$ for a given pure component, and we have then used a mass fraction $\left(m_{i}\right)$-based mixing rule (Equation 11) to estimate what PG might be required for a binary tablet of given weight $M$. The predicted PG values are in good agreement with the experimentally required values for binary tablets, with the difference being of a similar order of magnitude as tablet thickness (Fig. 3B). Such a relation is envisaged to be useful to users wishing to apply a certain compaction force.

$$
\begin{aligned}
& \gamma_{i}=a_{i} P^{b_{i}} \\
& P G=M \sum_{i \in C} m_{i} \gamma_{i}
\end{aligned}
$$



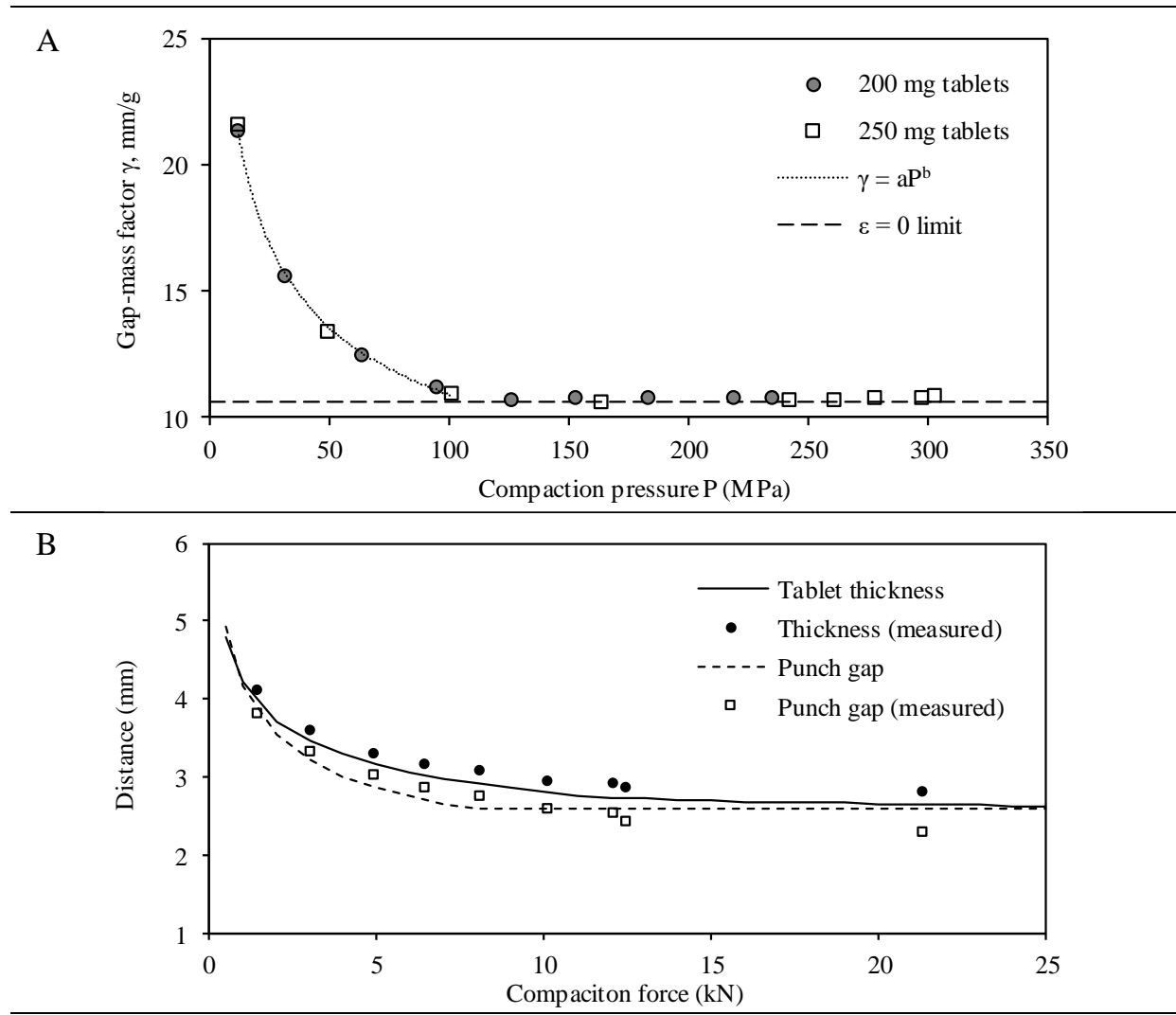

Figure 3. A) Punch gap-compaction pressure relation for Avicel ${ }^{\circledR} \mathrm{PH}-101$. Data points have been normalized by dividing punch gap values $(\mathrm{mm})$ by tablet weights $(\mathrm{g})$. B) Prediction from pure component data of required punch gap values of multicomponent tablets (Equations 10-11).

\section{Conclusions}

Pure component parameters can be fitted with good statistical results. The use of these pure parameters for the prediction of multicomponent tablet properties is possible, and predictions have been improved by a novel use of model equations. In addition, a relation between tablet press punch gap values and applied compaction pressures has been developed, which predicts necessary punch gaps with good accuracy.

\section{References}

Diab, S \& Gerogiorgis, DI, 2018. Ind. Eng. Chem. Res. 57, 9489-9499.

Fell, JT, Newton, JM, 1970. J. Pharm. Sci. 59, 688-691.

Gavi, E, Reynolds, GK, 2014. Comput. Chem. Eng. 71, 130-140.

Lee, SL, O'Connnor, TF, Yang, X, Cruz, CN, Chatterjee, S, Madurawee, RD, Moore, CMV, Yu, LX, Woodcock, J, 2015. J Pharm Innov. 10, 191-199.

Papathanasiou, F, 2018. (Masters Thesis). University of Strathclyde.

Reynolds, GK, Cambell, JI, Roberts RJ, 2017. Int J Pharm. 531, 215-224.

PSE, 2018. gPROMS Formulated Products. www.psenterprise.com (accessed 10.30.18).

Velasco, MV, Ford, JL, Rowe, P, Rajabi-Siahboomi, AR, 1999. J. Control. Release 57, 75-85. 